\title{
Efectividad de los métodos mixtos en investigación contextual en salud y educación
}

\author{
María Consuelo Sáiz-Manzanares ${ }^{1}$ y María-Camino Escolar- \\ Llamazares $^{1}$
}

\author{
1 Universidad de Burgos, España | mcsmanzanares@ubu.es | \\ https://orcid.org/0000-0002-1736-2089 | cescolar@ubu.es | \\ https://orcid.org/0000-0003-2448-0267
}

\begin{abstract}
Resumen: En las últimas décadas se está utilizando la metodología de investigación mixta en contextos naturales. Los diseños mixtos aúnan las ventajas de los métodos cuantitativos y cualitativos. Esto facilita la implementación de programas de intervención en contextos naturales. En este estudio, se presenta la utilización de métodos mixtos para el análisis de la percepción de los estudiantes de Ciencias de la Salud de la eficacia del uso en entornos Blended Learning de una metodología de Project Based Learning (PBL) con experiencias Flipped Classroom. Se utilizó un diseño mixto explicativo. Se analizaron datos cuantitativos, en una escala tipo Likert y cualitativos en preguntas abiertas. Estos últimos se procesaron aplicando una técnica de categorización con el software Atlas.ti v8, seguidamente se efectuó un análisis de frecuencias y de porcentajes en cada una de las categorías. Los resultados hallados con esta metodología ajusta la intervención a las necesidades reales del grupo de interés.
\end{abstract}

Palabras Clave: Flipped classroom; Blended learning; Project based learning; Diseños mixtos; Programas de intervención.

\section{Introducción}

En las últimas décadas en las investigaciones se están aplicando técnicas cuantitativas y cualitativas o la combinación de ambas en lo que se ha denominado métodos mixtos (Verd Pericás, 2016). Los diseños mixtos pretenden incorporar las ventajas de ambos métodos, con el fin de mitigar los inconvenientes que subyacen a cada uno de ellos, y a su vez incorporar sus ventajas en un proceso de investigación global (Reichardt, 1986). Por un lado, permiten profundizar en el análisis de todas las variables que inciden en una investigación y además otorgan un papel más activo a los sujetos con una construcción más dinámica de los modelos. Y, por otro lado, al utilizar elementos cuantitativos facilitan una mejor generalización y control de la investigación.

En general, se puede decir que los diseños mixtos registran y analizan datos tanto cuantitativos como cualitativos con el objetivo de llegar a entender un problema desde un prisma multidimensional manteniendo la fiabilidad y la validez (Creswell \& Plano Clark, 2007; Martínez, Castellano, \& Chacón, 2014; Plano Clark \& Ivankova, 2016). En este sentido, los diseños mixtos son útiles cuanto existen problemas complejos, con múltiples facetas y realidades, necesitando de datos cualitativos y cuantitativos para cubrir todas ellas (Martínez et al., 2014). La convergencia de resultados entre ambos tipos de datos puede suponer una alta validez del estudio, pues redunda en la seguridad de las conclusiones (Chacón-Moscoso et al., 2019; Preciado, Anguera, Olarte, \& Lapresa, 2019)

El único inconveniente es que son más costosos en su aplicación (Martínez et al., 2014), pues en general suponen un mayor gasto tanto económico como de tiempo y esfuerzo. Un diseño mixto con varias etapas secuenciales cualitativo-cuantitativo complicará las etapas del diseño, de recogida de datos y de análisis (Escolano-Pérez, Herrero-Nivela, \& Anguera, 2019). Igualmente, no todos los diseños mixtos pueden llevarse a cabo, antes de empezar es necesario determinar si el diseño mixto es el más adecuado o si será posible realizarlo (Alcover et al., 2019).

Por otra parte, se han identificado diversos escenarios metodológicos en los que los diseños mixtos son de especial relevancia. 
Por ejemplo, para realizar comparaciones entre resultados cualitativos y cuantitativos y de esta forma comparar la coherencia entre ambos tipos de datos (Yauch \& Steudel, 2003). Para validar datos cuantitativos, por ejemplo, utilizando algunas preguntas abiertas al final de un cuestionario. Para mejorar un estudio experimental (Donovan et al., 2002), o para explicar relaciones en estudios correlacionales (Harrison, 2005; Martínez et al., 2014). Igualmente, se pueden utilizar datos cuantitativos para cribar masivamente posibles participantes a los que después se someterá a alguna recogida de datos cualitativos en profundidad (entrevistas o análisis telemáticos) (Martínez et al., 2014). De la misma forma, se pueden utilizar datos cuantitativos para identificar cuestiones de interés que luego se utilizaran en un cuestionario cuantitativo (Mark \& Marshall, 2004; Martínez et al., 2014).

En este sentido, el uso de dichos métodos es especialmente relevante en los contextos educativos y de la salud, ya que el análisis de información situacional es esencial en la aplicación acertada de técnicas o programas de intervención o de tratamiento. Actualmente, gran parte de los estudios se realizan con una metodología mixta que aplica:

a) Técnicas de recogida de información directa. Implica el registro de conductas en situaciones naturales, para ello habitualmente se utilizan grabaciones en vídeo audio y siendo el observador participante (Sáiz \& Escolar, 2013).

b) Técnicas de recogida de información indirecta. Estas se realizan a través documentación en vídeo o en audio sobre acciones en las que el observador no es participante (Banks, 2010; Gibbs, 2012). Muchas de estas técnicas se han incorporado recientemente al acervo metodológico (Del Giacco, Anguera, \& Salcuni, 2020).

Tanto, las técnicas de recogida de información directa como indirecta permiten incluir recursos hipermedia e informáticos que facilitan tanto el registro como la transformación de la información para su posterior análisis (Queiruga \& Sáiz, 2016; Marticorena, Sáiz, Arnaiz, Escolar, \& Queiruga, 2018; Sáiz, García-Osorio, \& Díez-Pastor, 2019). En este contexto de investigación, la categorización y codificación de la información es fundamental ya que el estudio en contextos naturales proporciona al investigador un gran volumen de datos (Sáiz, Marticorena, Arnaiz-González, Díez-Pastor, \& Rodríguez-Arribas, 2019a; Sáiz \& Román, 2012). Dichos datos pueden o no ser relevantes para el objeto de estudio, por ello el investigador tiene que delimitar a priori de forma clara dicho objeto. Este hito le permitirá aplicar un buen proceso de categorización de la información registrada (Anguera, 1986, 1991). Todo ello, facilita el trabajo con matrices de datos. Así como la aplicación de técnicas novedosas en el análisis de los datos observacionales. Por ejemplo, a través del análisis de coocurrencias ${ }^{1}$ y ocurrencias, del Análisis Secuencial de Retardos ${ }^{2}$ (ASR), del análisis de coordenadas polares ${ }^{3}$, de los T-patterns ${ }^{4}$, y del análisis de grafos ${ }^{5}$, etc., entre otros.

\footnotetext{
1 Indica el estudio cruzado de las frecuencias en las categorizaciones realizadas.

2 "El análisis secuencial de retardos es una de las modalidades de análisis secuencial, y tiene como objetivo último la detección de patrones secuenciales de conducta, lo cual se lleva a cabo mediante la búsqueda de contingencias secuenciales entre categorías o códigos de conducta (según que el instrumento de observación utilizado haya sido el sistema de categorías o los formatos de campo, respectivamente)" (Anguera, 2014, p.19).

3 "Es una técnica de reducción drástica de datos basada en el Zsum de Cochran (1954), implementada por Sackett (1980) y optimizada con la "técnica genuina" por Anguera (1997). Tiene como objetivo la vectorialización del comportamiento y el establecimiento de las relaciones entre una conducta focal y el resto de categorías del sistema" (Anguera, 2014, p.17).

${ }^{4}$ Descubrimiento de estructuras ocultas que conforman la realidad del comportamiento observado, pero que no resultan directamente visibles" p.20 Anguera (2014).

5 También denominada análisis de redes, es una técnica computacional que analiza las relaciones entre los elementos o variables, que aplica técnicas de optimización como son la probabilidad o la combinatoria desde la aplicación de algoritmos.
} 
Además, en la última década se han incluido en el procesamiento de los datos la aplicación de técnicas de Minería de Datos o Data Mining (Slater, Joksimović, Kovanovic, Baker, \& Gasevic, 2016) tanto de aprendizaje supervisado, como no supervisado (Sáiz, Marticorena, Arnaiz-González, Díez-Pastor, \& Rodríguez-Arribas, 2019b; Sáiz, Marticorena, Arnaiz-González, Escolar, \& Queiruga-Dios, 2018). Las técnicas de aprendizaje supervisado pretenden descubrir las relaciones entre los atributos de entrada (variables o características) y un atributo de destino (clase) o supervisor. En el aprendizaje no supervisado, no existe supervisor y solo hay datos de entrada disponibles (Sáiz, Marticorena et al., 2019b). El objetivo en este caso es encontrar regularidades, irregularidades, relaciones, similitudes y asociaciones en la entrada (Sáiz, Queiruga, García-Osorio, Montero, \& Rodríguez-Medina, 2019). Asimismo, en el análisis de datos no hay que olvidar la aplicación de técnicas más tradicionales como son las que ofrece la estadística descriptiva, el análisis de correspondencias, el análisis de la varianza, el análisis de regresión, etc. (Sáiz, García-Osorio, \& Díez-Pastor, 2019). Es decir, la aplicación de técnicas analíticas en los diseños observacionales, tal y como plantean autores como Anguera, Blanco y Losada (2001).

En este escenario, la formación de futuros investigadores en el manejo de los diseños de investigación mixtos y en la utilización fluida de técnicas mixtas de análisis de datos, es una necesidad cada vez más apremiante, tal y como se reflejan en trabajos como los de Sáiz y Montero (2016). En resumen, la investigación tiene el reto de ser aplicada y sostenible en el marco de una sociedad global en continua evolución tanto en objetivos como en la incorporación de novedades tecnológicas que facilitan su desarrollo pero que confieren una responsabilidad de formación y aplicación para los profesionales de la educación y de la salud.

Seguidamente, se van a abordar de forma sucinta las etapas en la realización de un diseño mixto y los tipos de diseño más representativos.

\subsection{Etapas en la realización de un diseño mixto}

Las etapas para la realización de un diseño mixto se deben abordar de una forma flexible ya que existe una interrelación entre ellas (Martínez et al., 2014). Dichos hitos son:

1. Identificar el problema y analizar las características que el objeto de la investigación tiene.

2. Estudiar qué partes o elementos del problema pueden ser abordados desde un enfoque cuantitativo y cuáles desde un enfoque cualitativo.

3. Identificar qué información cuantitativa y cualitativa es necesario recoger.

4. Analizar los datos. Debe estudiarse qué información aportará cada parte, en qué formato se codificará y si es posible convertirla a un formato común.

5. Elegir el método mixto más adecuado. En función de las etapas anteriores se eligirá el diseño mixto más apropiado a las necesidades del trabajo de investigación.

6. Diseñar el cronograma. La recogida de la información dentro de un diseño mixto suele ser compleja, por lo que es necesaria una estricta planificación de las etapas de recogida y análisis. Para ello, se pueden utilizar diagramas de Gantt.

7. Realizar la investigación. Estas etapas de recogida y análisis son similares a otras investigaciones (Martínez et al., 2014).

\subsection{Tipos de diseños mixtos}

Se pueden distinguir cuatro tipos de diseños mixtos (Creswell \& Plano Clark, 2007; Martínez et al., 2014). 


\subsubsection{Diseño Explicativo}

Este diseño es una ampliación directa de un método cuantitativo. El objetivo es el de obtener información cuantitativa, utilizando posteriormente una etapa cualitativa para explicar, potenciar o refinar los resultados obtenidos en la anterior. Este tipo de diseño no conlleva integración de datos, cada tipo de datos se analiza por separado integrándose los resultados de ambos en una interpretación global al final del estudio (Creswell \& Plano Clark, 2007; Martínez et al., 2014).

\subsubsection{Diseño exploratorio}

Este diseño es secuencial al igual que el anterior el orden de la secuencia es invertida. Primero se realiza la etapa cualitativa y después la cuantitativa (Creswell \& Plano Clark, 2007; Martínez et al., 2014).

\subsubsection{Diseño de triangulación}

En este diseño las dos etapas se diseñan y se recogen los datos en paralelo, para posteriormente llegar a una interpretación conjunta. No existe una jerarquía o subordinación de una parte sobre la otra y ambas contribuyen con la misma importancia a las conclusiones (Creswell \& Plano Clark, 2007; Martínez et al., 2014).

\subsubsection{Diseño integrado (embedded design)}

En este diseño los datos también se recogen de forma concurrente, aunque hay una jerarquía entre ellos, estando un tipo de datos subordinado al otro, con el objetivo de potenciar y dar apoyo a los primeros. Es importante que la relación entre los datos secundarios y primarios sea lógica y clara, que hay una convergencia y que quede bien demostrado que los segundos confirman parte de los primeros (Creswell \& Plano Clark, 2007; Martínez et al., 2014).

\section{La investigación / método / el proyecto}

En esta sección primero se abordará el proceso de investigación, seguidamente el método aplicado y finalmente el desarrollo del proyecto.

\subsection{La investigación}

En este estudio se ha realizado un análisis de la percepción de los estudiantes de dos Grados en Ciencias de la Salud: Terapia Ocupacional y Enfermería, respecto de la práctica docente. Dicha práctica se realizó en un entorno Blended Learning en el que se aplicó una metodología de Project Based Learning ${ }^{6}$ (PBL).

El diseño de investigación fue mixto explicativo, lo que permitió obtener, en primer lugar, información cuantitativa, para posteriormente refinar los resultados com información cualitativa.

${ }^{6}$ Es una metodología de enseñanza-aprendizaje que se basa en la aplicación de conceptos a supuestos prácticos mediante el cual los alumnos desarrollan competencias en un enfoque colaborativo. 
Este diseño permite integrar ambos resultados en una interpretación global.

Asimismo, se abordó la pregunta de investigación «¿Qué grado de utilidad tiene para los estudiantes las acciones Flipped Classroom ${ }^{7}$ en entornos Blended Learning ${ }^{8}$ ?». Las variables fueron: La utilidad percibida por parte de los estudiantes de la metodología Flipped Classroom sobre la comprensión conceptual y procedimental en entornos de aprendizaje Blended Learning.

\subsection{Fases del proceso / de la investigación}

Antes de iniciar el estudio se informó a los estudiantes los objetivos del mismo, su participación fue voluntaria y se recabó su compromiso informado por escrito y se obtuvo el informe positivo del Comité de Bioética de la Universidad de Burgos. En este estudio se trabajó con una metodología PBL en entornos de aprendizaje Blended Learning que se desarrollaron en una plataforma interactiva, UBUVirtual, basada en Moodle (Modular Object-Oriented Dynamic Learning Environment). En este entorno se utilizaron recursos hipermedia dentro de experiencias Flipped Classroom. Los dos últimos temas en las dos asignaturas (una en el Grado en terapia Ocupacional y otra en el Grado en Enfermería) aplicaron estas experiencias para lo que se utilizaron vídeos. Dichos vídeos incluían preguntas de orientación tipo test (quizzes). Las asignaturas seguían el siguiente procedimiento: en cada unidad temática se realizaba una exposición en el aula de los contenidos teóricos (clase presencial). Asimismo, en cada unidad había una actividad práctica relacionada directamente con los conceptos teóricos vistos en la clase presencial. Al finalizar la explicación del tema, el estudiante realizaba un cuestionario de evaluación, en la plataforma, sobre los conocimientos tratados. Una vez concluido dicho cuestionario, cada alumno recibía un feedback sobre las respuestas dadas y si estas eran correctas o incorrectas y por qué en ambos casos. De igual modo, al finalizar cada unidad temática el estudiante rellenaba una encuesta de evaluación sobre el desarrollo de la misma. El objetivo era el de recibir un feedback sobre la percepción del desarrollo del proceso de enseñanza. Todos los cuestionarios se realizaron desde la plataforma UBUVirtual.

El diseño de la asignatura contemplaba acciones sincrónicas [trabajo en el tiempo presencial en trabajo de los conceptos y actividades de Gran Grupo (GG) y de Pequeño Grupo (PG), desde la metodología de PBL] y acciones asincrónicas (trabajo en PG fuera del aula para la elaboración del proyecto la realización de las prácticas, la visualización realización de las actividades de evaluación y de reflexión sobre los vídeos que sirvieron de apoyo y de refuerzo a la acción formativa). La retroalimentación el docente, como ya se ha indicado fue de feedback orientado a procesos e individualizado para cada estudiante.

\subsection{Instrumentos}

\section{a) Flipped Classroom}

Se utilizaron experiencias Flipped Classroom que consistieron en vídeos elaborados ad hoc por la profesora. Dichos vídeos estaban a disposición de los estudiantes antes del desarrollo de la clase presencial. En la Figura 1 se presenta la estructura de trabajo en la plataforma UBUVirtual de las experiencias Flipped Classroom.

En la asignatura 1 se utilizaron los siguientes vídeos cuya referencia se puede consultar en el apartado de referencias:

\footnotetext{
${ }^{7}$ Es una metodología de enseñanza-aprendizaje que se basa en la realización de experiencias de aprendizaje en entornos distintos al aula presencial, fundamentalmente se realiza a través de vídeos en los que se trabajan conceptos que después se aplican de una forma práctica en el aula presencial.

$8 \mathrm{O}$ aprendizaje mixto, implica que el proceso de enseñanz-aprendizaje se realiza de forma presencial y no presencial, es decir a través de interacciones en plataformas virtuales (Learning Management System).
} 
Vídeo Unidad Temática 4. Hace referencia a conceptos sobre desarrollo evolutivo 0-3 años y sobre técnicas de intervención temprana en estas edades.

Vídeo Unidad Temática 5. Hace referencia a conceptos sobre desarrollo evolutivo 3-6 años y sobre técnicas de intervención temprana en estas edades.

En la asignatura 2 se utilizó el vídeo:

Vídeo Unidad Temática 4 y 5 . Hacen referencia a conceptos de metodología de investigación en calidad de servicios en enfermería.

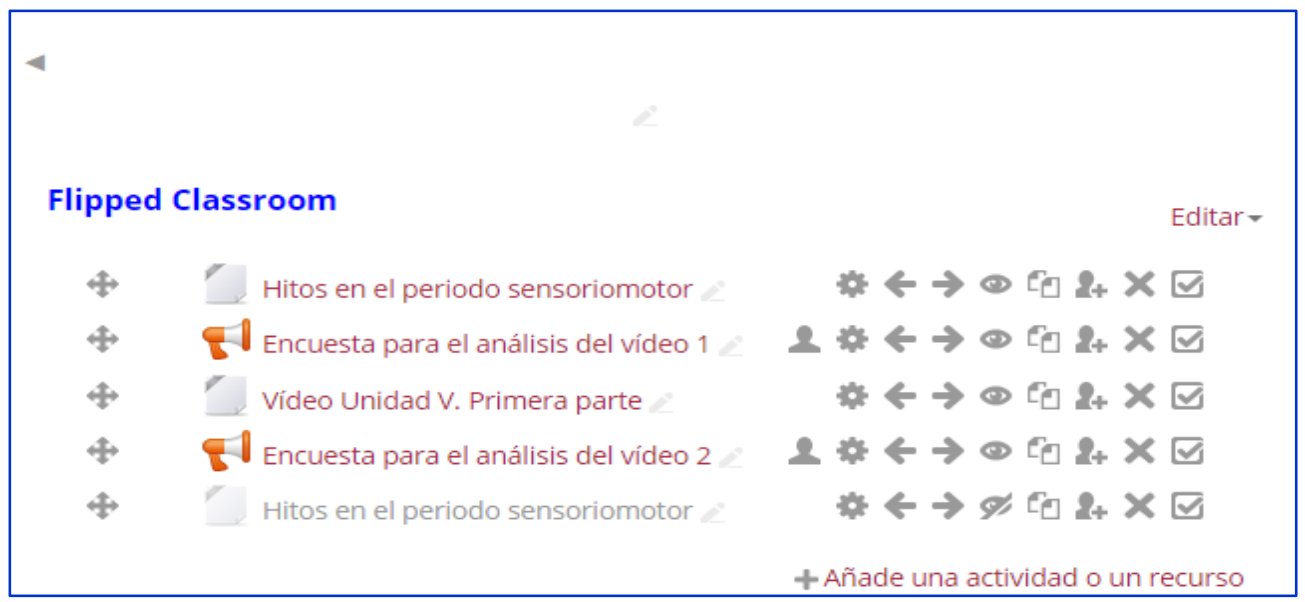

Fig. 1. Estructura en la plataforma de la experiencia Flipped Classroom.

b) Cuestionario de valoración de la experiencia Flipped Classroom

Dicho instrumento consta de 4 preguntas, 1 de opción múltiple en una escala tipo Likert de 1 a 5 , desde nada (1), hasta todo (5), sobre la utilidad del vídeo en la comprensión de los contenidos de la unidad correspondiente. $Y$ tres preguntas abiertas relacionadas con: la utilidad de la experiencia, aspectos a ampliar y a eliminar. Al tener un solo ítem de respuesta múltiple no se pudo hallar el coeficiente de fiabilidad.

\subsection{Participantes}

Se trabajó con una muestra de 67 alumnos de tercero de grado en Titulaciones de Ciencias de la Salud, 45 en el Grado en Terapia Ocupacional (GTO) y 22 en el Grado en Enfermería (GE) en la Tabla 1 se presentan los estadísticos descriptivos atendiendo a la variable sexo.

Tabla 1. Distribución de los grupos y variables de Media y DT en la variable edad

\begin{tabular}{llllllll}
\hline Grupo & \multicolumn{4}{c}{ Hombres } & \multicolumn{3}{c}{ Mujeres } \\
\hline & $\mathrm{N}$ & $n$ & $M_{\text {edad }}$ & $D T_{\text {edad }}$ & $n$ & $M_{\text {edad }}$ & $D T_{\text {edad }}$ \\
GTO & 45 & 4 & 24 & 2.82 & 23.5 & 6.17 & 23.5 \\
GE & 22 & 3 & 22.3 & 2.30 & 19 & 21.8 & 1.22 \\
\hline
\end{tabular}

Nota. $M_{e d a d}=$ Media de la variable edad; $D T_{e d a d}=$ Desviación Típica de la variable edad . 


\section{Análisis de datos}

Se analizaron las respuestas de los estudiantes a la encuesta de opinión. Para ello, primero se realizó un análisis de porcentajes a la pregunta cerrada que se había medido en una escala tipo Likert de 1 a 5 , donde 1 implicaba "ninguna utilidad" y 5 = "mucha utilidad". En segundo lugar, se analizaron los datos recogidos en las preguntas abiertas. Para ello, primero se efectuó una categorización de las respuestas. Dicha categorización se realizó con la ayuda del software Atlas.ti v.8. Un ejemplo del proceso de categorización, realizado ad hoc, se puede consultar en la Figura 2.

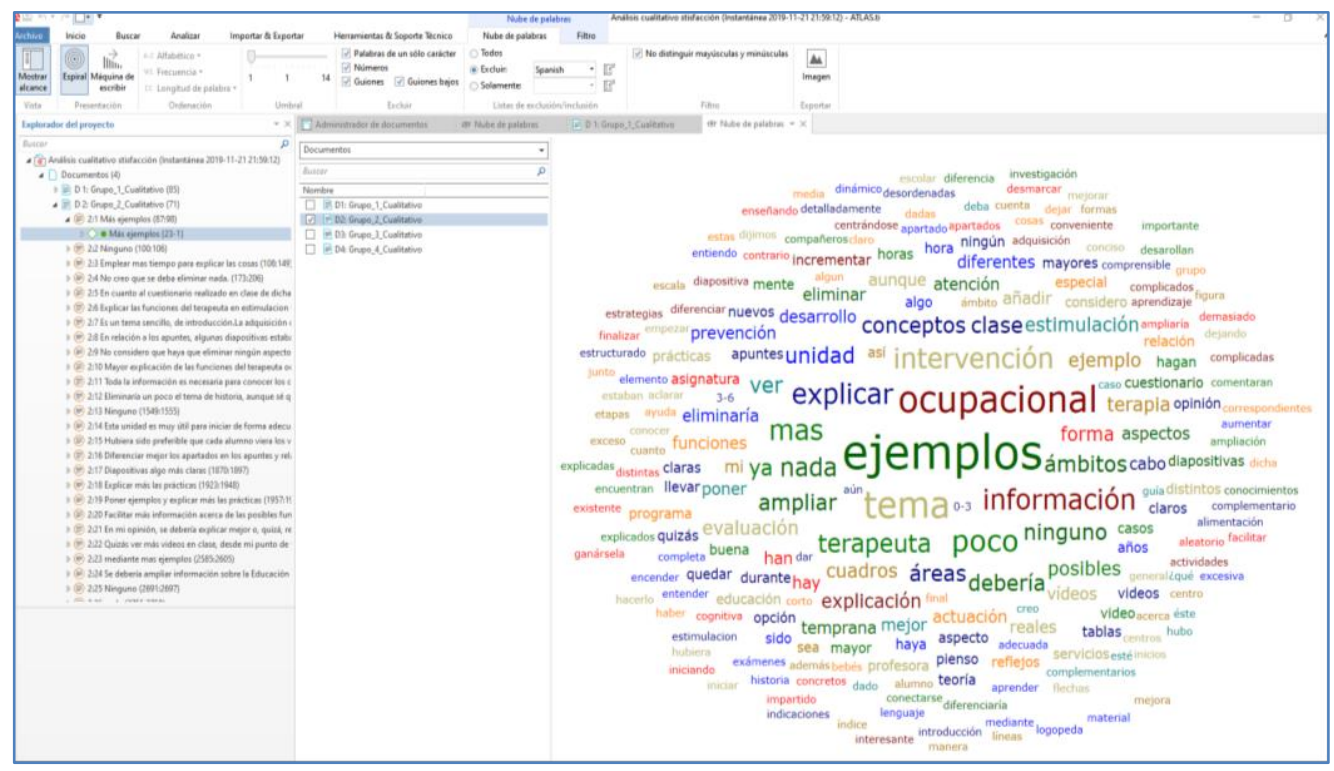

Fig. 2. Proceso de categorización de las respuestas a las preguntas abiertas con el programa Altlas.ti v.8.

Seguidamente las frecuencias halladas en cada una de las categorías se exportaron a una hoja Excel. Dicha hoja a su vez se exportó al software SPSS v.24 en el que se realizó un análisis de porcentajes (en la Figura 3 se presenta un esquema gráfico del proceso).

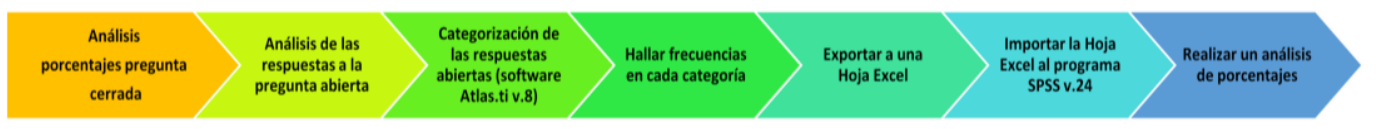

Fig. 3. Esquema gráfico del proceso de análisis de datos.

\section{Resultados}

\subsection{Resultados en la pregunta cerrada tipo Likert}

Se analizaron las respuestas de los estudiantes de ambos grados a la pregunta cerrada « ¿La explicación de los conceptos clave de la unidad en el vídeo han facilitado la comprensión?». En la Figura 4 y en la Figura 5 se presentan los porcentajes de satisfacción de los estudiantes con la utilización de la experiencia Flipped Classroom. 
Como se puede observar los estudiantes, en ambos grados, percibieron la utilización de esta metodología como facilitadora de la comprensión de las unidades temáticas.

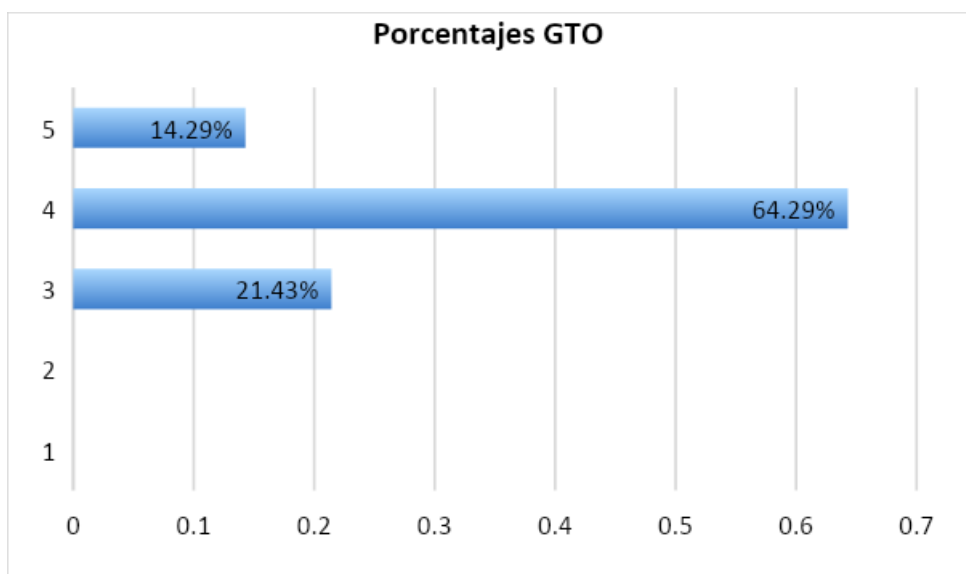

Fig. 4. Porcentaje de acuerdo con el ítem: «Los conceptos clave trabajados en el vídeo me han facilitado la comprensión del tema» en el Grado en Terapia Ocupacional (GTO). La escala de gradación va desde 1 = ninguna utilidade, hasta 5 = Muy útil.

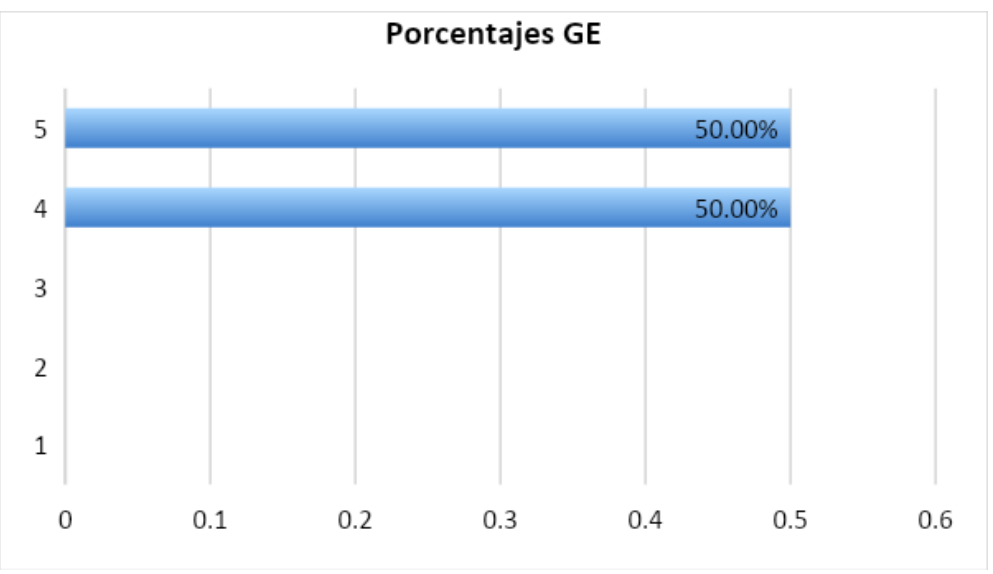

Fig. 5. Porcentaje de acuerdo con el ítem: «Los conceptos clave trabajados en el vídeo me han facilitado la comprensión del tema» en el Grado en Enfermería (GE). La escala de gradación va desde 1 = ninguna utilidad hasta $5=$ Muy útil.

\subsection{Resultados en las preguntas abiertas}

Respecto de la pregunta abierta («¿Qué aspectos incrementarías en un espacio Flipped Classroom»)? que nos permite realizar un análisis cualitativo a nivel descriptivo, los estudiantes señalaron que sería necesario incrementar los mapas conceptuales, los ejemplos, y las pruebas tipo quizzes ${ }^{9}$ (ver Figura 6 y Figura 7). Relativo a los aspectos a eliminar ambos grupos coincidieron en que no eliminarían ninguno de los recursos aplicados en las experiencias Flipped Classroom. Un ejemplo de las respuestas dadas por los estudiantes y la categorización realizada se puede consultar en el Apéndice 1.

${ }^{9}$ Son preguntas que se realizan en cuestionarios y pueden ser de varios tipos de opción de respuesta múltiple o de opción de respuesta dicotómica. 


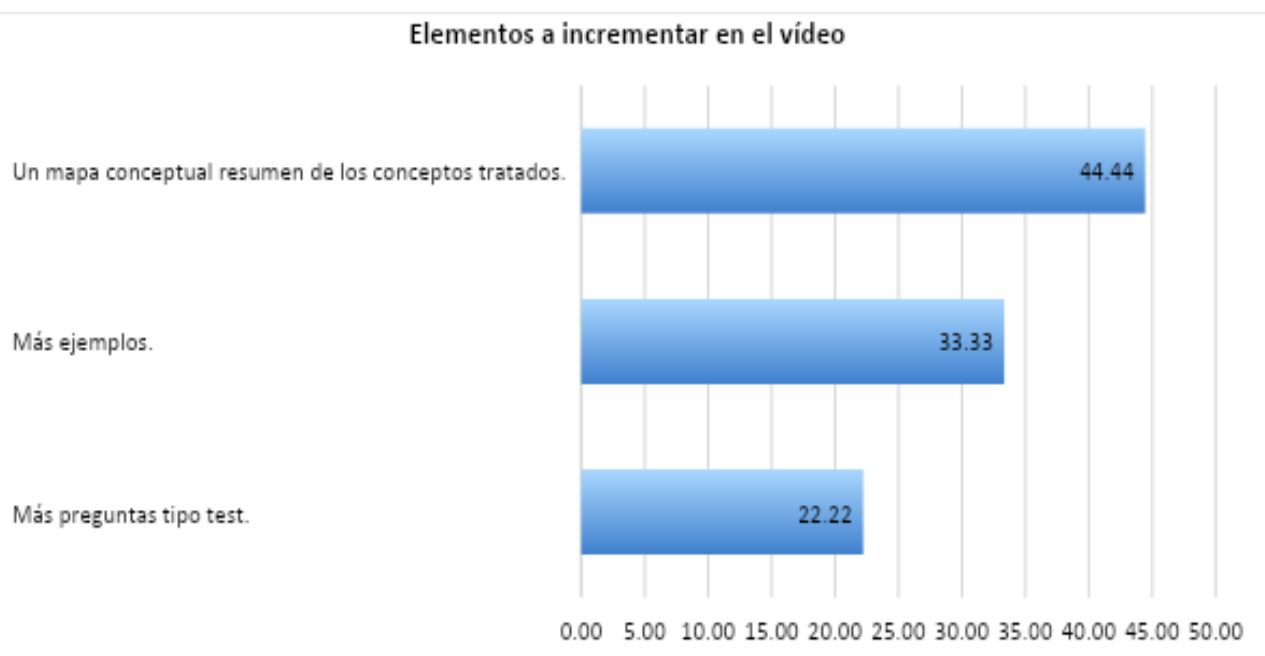

Fig. 6. Porcentaje de los elementos a incrementar en el vídeo señalados por los estudiantes del Grado en Terapia Ocupacional.

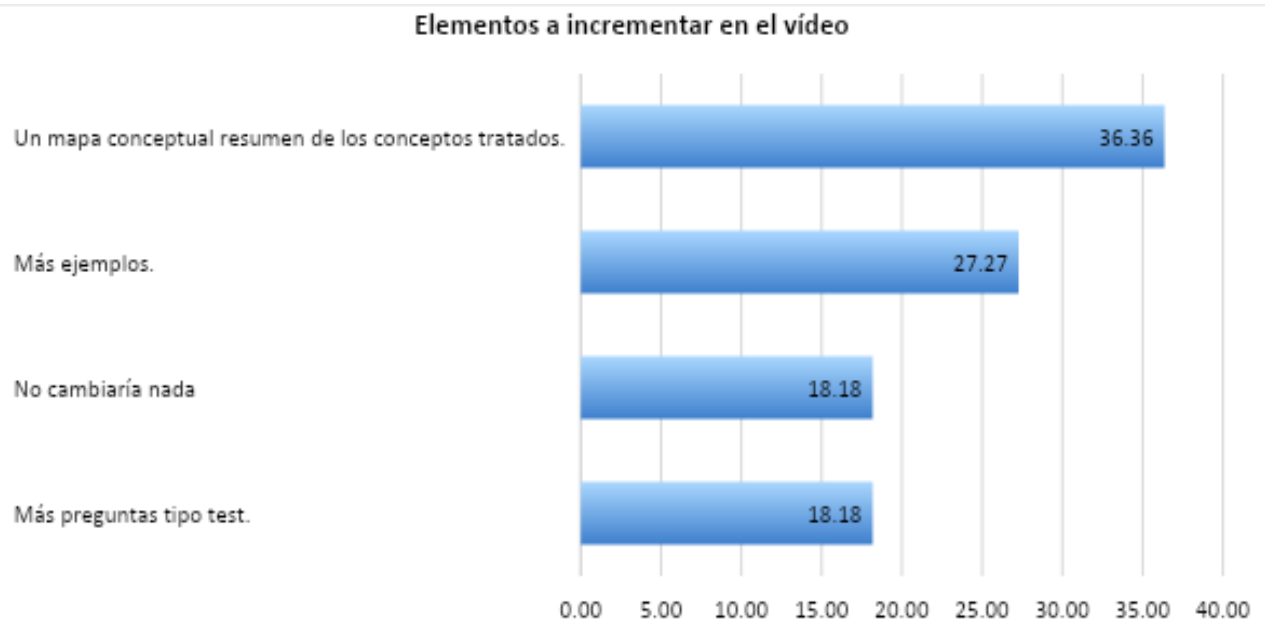

Fig. 7. Porcentaje de los elementos a incrementar en el vídeo señalados por los estudiantes del Grado en Enfermería.

\section{Guidelines para estudios siguientes}

Las conclusiones de este estudio tienen que ser examinadas con prudencia respecto de la generalización de los resultados, debido al tamaño de la muestra y al origen de la misma, estudiantes de una misma universidad en dos titulaciones. Por ello, estudios posteriores irán dirigidos a ampliar la muestra en diferentes poblaciones de estudiantes universitarios que utilicen la plataforma Moodle en distintas titulaciones.

\section{Aplicabilidad en otros contextos}

La investigación que se ha presentado en este capítulo ha pretendido dar información acerca de la aplicabilidad de las técnicas de investigación mixta en contextos de instrucción en estudiantes de Ciencias de la Salud. Asimismo, dicho trabajo ha aportado ideas e instrumentos para su aplicación en otras realidades educativas en contextos 
universitarios (Sáiz, Escolar, Marticorena, García-Osorio, \& Queiruga, 2017a; Sáiz, Escolar, Marticorena, García-Osorio, \& Queiruga, 2017b). Este hecho será esencial en los procesos de transferencia y generalización de las experiencias de investigación y darán luz para abordar futuras líneas de trabajo en colaboración con otros profesionales e instituciones (Sáiz, Escolar, Marticorena, García-Osorio, \& Queiruga, 2017c).

\section{Conclusiones}

El desarrollo de la metodología Flipped Classroom en espacios de aprendizaje Blended Learning es actualmente una herramienta relevante en los contextos de formación de estudiantes en la rama de Ciencias de la Salud. Uno de los logros de la misma es que facilita el feedback del docente prácticamente en tiempo real y posibilita la individualización del aprendizaje. De hecho los estudiantes han valorado estas experiencias como un elemento esencial que les ayuda a aprender mejor.

En síntesis, la metodología Flipped Classroom en entornos Blended Learning ayuda a crear entornos de aprendizaje activos, ya que combina la instrucción presencial y la instrucción vía tecnológica y da ayudas instruccionales en ambos entornos, tanto al acceso al conocimiento, como a su aplicación.

Estudios posteriores se orientarán a la elaboración de programas en línea para la tutorización y mentorización individualizada de los alumnos, así como para la extracción de sus perfiles de aprendizaje en tiempo real en las plataformas de aprendizaje como Moodle.

Finalmente se puede concluir señalando la aplicabilidad de la metodología de métodos mixtos al análisis del proceso de enseñanza-aprendizaje en contextos naturales de enseñanza universitaria en los que se aplican metodologías activas y recursos hipermedia. El análisis de la percepción de los estudiantes sobre la efectividad de esta metodología desde el uso de métodos mixtos ayuda en la especificación clara de las necesidades de los estudiantes y orienta al docente sobre la respuesta educativa más adecuada en cada caso (tanto grupal como individual).

\section{Referencias}

Alcover, C., Mairena, M. Á., Mezzatesta, M., Elias, N., Díez-Juan, M., Balañá, G., ... Arias-Pujol, E. (2019). Mixed methods approach to describe social interaction during a group intervention for adolescents with autism spectrum disorders. Frontiers in Psychology, 10(JUN), 1-14. https://doi.org/10.3389/fpsyg.2019.01158

Anguera, M.T. (1986). La investigación cualitativa. Educar, 10, 23-50. Recuperado de https://educar.uab.cat/article/viewFile/v10-anguera/442

Anguera, M.T. (1991). Metodología Observacional en la investigación Psicológica (Vol. 1). Barcelona: PPU.

Anguera, M. T., Blanco, A., \& Losada, J. L. (2001). Diseños observacionales, cuestión clave en el proceso de la Metodología Observaciones. Metodología de las Ciencias del Comportamiento 3(2), 135-160.

Banks, M. (2010). Los datos visuales en investigación cualitativa. Madrid: Morata.

Chacón-Moscoso, S., Anguera, M. T., Sanduvete-Chaves, S., Losada, J. L., Lozano-Lozano, J. A., \& Portell, M. (2019). Methodological quality checklist for studies based on observational methodology (MQCOM). Psicothema, 31(4), 458-464. https://doi.org/10.7334/psicothema2019.116

Creswell, J. W., \& Plano Clark, V.L. (2007). Designing and conducting mixed methods research. Thousand Oaks, CA: Sage

Del Giacco, L., Anguera, M.T., \& Salcuni, S. (2020). The Action of Verbal and Non-verbal Communication in the Therapeutic Alliance Construction: A Mixed Methods Approach to 
Assess the Initial Interactions With Depressed Patients. Frontiers in Psychology, 11(234). https://doi.org/10.3389/fpsyg.2020.00234

Donovan, J., Mills, N., Smith, M., Brindle, L., Jacoby, A., Peters, T., et al. (2002). Improving desing and conduct of randomized trials by embedding them in qualitative research: ProtedT (prostate testing for cancer and treatment) study. British Medical Journal, 325, 766-769.

Escolano-Pérez, E., Herrero-Nivela, M. L., \& Anguera, M. T. (2019). Preschool metacognitive skill assessment in order to promote educational sensitive response from mixed-methods approach: Complementarity of data analysis. Frontiers in Psychology, 10(JUN), 1-22. https://doi.org/10.3389/fpsyg.2019.01298

Gibbs, G. (2012). El análisis de datos cualitativos en Investigación Cualitativa. Madrid: Morata

Harrison, A. (2005). Correlates of positive relationship building in a teacher education mentoring program (Tesis doctoral). University of Nebraska, Lincoln (capítulo 12).

Queiruga, M.A., \& Sáiz, M.C. (2016). Análisis de Protocolos en alumnos de Educación Secundaria Obligatoria: un análisis del pensamiento metacognitivo en la asignatura de Física (Tesis Doctoral no publicada). Universidad de Burgos.

Mak, L., \& Marshall, S.K. (2004). Perceived mattering in young adults'romantic relationships. Journal of Social and Personal Relationships, 24(4), 469-486

Marticorena, R., Sáiz., M.C., Arnaiz, Á., Escolar, M.C., \& Queiruga, M.A. (2018). Análisis de los resultados de aprendizaje en Ciencias de la Salud: Learning Analytics desde un plugin para Moodle. En M. Molero et al. (coord.), Intervención en Contextos Clínicos y de la Salud (Vol. II, pp. 243-252). Almería: ASUNIVEP.

Martínez, R., Castellano, M.A., \& Chacón, J.C. (2014). Métodos de investigación en psicología. Madrid: EOS Universitaria.

Plano Clark, V.L., \& Ivankova, N.V. (2016). Mixed Methods Research: A Practical Guide to the Field. Thousand Oaks: Sage.

Preciado, M., Anguera, M. T., Olarte, M., \& Lapresa, D. (2019). Observational studies in male elite football: A systematic mixed study review. Frontiers in Psychology, 10(OCT). https://doi.org/10.3389/fpsyg.2019.02077

Reichardt, Ch.S. (1986). Hacia una superación del enfrentamiento entre los métodos cualitativos y los cuantitativos. En T.D. Cook and Ch.S. Reichardt (Eds.), Métodos cualitativos y cuantitativos en investigación evaluativa (pp. 25-58). Madrid: Morata.

Sáiz, M.C., \& Escolar, M.C. (2013). Observación sistemática e investigación en contextos educativos. Burgos: Servicio de Publicaciones de la Universidad de Burgos.

Sáiz., M.C., Escolar, M.C., Marticorena, R., García-Osorio, C.I., \& Queiruga, M.A. (2017a). Aprendizaje basado en proyectos utilizando LMS: una experiencia en Ciencias de la Salud. En J.C Núñez., et al. (Eds.), Temas actuales de investigación en las áreas de la Salud y la Educación (pp. 739-746). Oviedo: SCINFOPER

Sáiz., M.C., Escolar, M.C., Marticorena, R., García-Osorio, C.I., \& Queiruga, M.A (2017b) Conductas de aprendizaje en LMS, SRL y feedback efectivo en B-Learning. En J.C Núñez. et al. (Eds.), Temas actuales de investigación en las áreas de la Salud y la Educación (pp. 747752). Oviedo: SCINFOPER

Sáiz, M.C., Escolar, M.C., Marticorena, R., García-Osorio, C.I., \& Queiruga, M.A. (2017c) Formación del profesorado en Metodologías Activas desde Plataformas interactivas. En J.C Núñez. et al. (Eds.), Temas actuales de investigación en las áreas de la Salud y la Educación (pp. 39-44). Oviedo: SCINFOPER

Sáiz, M.C., García-Osorio, C.I., \& Díez-Pastor, J.F. (2019). Differential efficiency of the resources used in b-learning environments. Psicothema, 31(2), 170-178. doi: 10.7334/psicothema2018.330

Sáiz, M.C., Queiruga, M.Á., García-Osorio, C.I., Montero, E., \& Rodríguez-Medina, J. (2019) Observation of Metacognitive Skills in Natural Environments: A Longitudinal Study with Mixed Methods. Frontiers in Psychology, 10(2398), 1-13. doi: 10.3389/fpsyg.2019.02398 
Sáiz, M.C., Queiruga-Dios, M.Á., Marticorena, R., Escolar, M.C., \& Arnaiz-González, Á. (2018). Cuestionarios de e-autoevaluación y e-feedback: una aplicación en Moodle. European Journal of Health Research, 4(3), 135-148. doi: 10.30552/ejhr.v4i3.116

Sáiz, M.C., Marticorena, R., Arnaiz-González, Á., Escolar, M.C., \& Queiruga-Dios, M.Á. (2018). Detección del alumno en riesgo en titulaciones de Ciencias de la Salud: aplicación de técnicas de Learning Analytics. European Journal of Investigation in Health, Psychology and Education, 8(3), 129-142. doi: 10.30552/ejihpe.v8i3.273

Sáiz, M.C., Marticorena, R., Arnaiz-González, Á., Díez-Pastor, J.F., \& Rodríguez-Arribas, S. (2019a). Computer application for the registration and automation of the correction of a functional skills detection scale in Early Care. 13th International Technology, Education and Development Conference Proceedings of INTED2019 Conference 11th-13th March 2019 (pp. 5322-5328).

Sáiz, M.C., Marticorena, R., Arnaiz-González, Á., Díez-Pastor, J.F., \& Rodríguez-Arribas, S. (2019b). Blended learning: an experience with infographics and virtual laboratories using Self-regulated learning. 13th International Technology, Education and Development Conference Proceedings of INTED2019 Conference 11th-13th March 2019 (pp. 2966-2971). IATED: Valencia (Spain).

Sáiz, M.C., \& Montero, E. (2016). Metodologías activas en docencia universitaria. Diseño de una asignatura de ciencias de la salud en la plataforma virtual. Burgos: Servicio de Publicaciones de la Universidad de Burgos.

Sáiz, M.C., \& Román, J.M. (2012). Estimulación temprana en una alumna con Síndrome de Down. Revista de Audición y Lenguaje, 97(6), 14-21.

Slater, S., Joksimović, S., Kovanovic, V., Baker, R. S., \& Gasevic, D. (2016). Tools for Educational Data Mining: A Review. Journal of Educational and Behavioral Statistics, 42(1), 85-106. doi: 10.3102/1076998616666808

Verd Pericás, J.M. (2016). Introducción a la investigación cualitativa. Madrid: Síntesis.

Yauch, C.A., \& Steudel, H.J. (2003). Complementary use of qualitative and quantitative cultural assessment methods. Organizational Research Methods, 6(4), 465-481.

\section{Vídeos}

Asignatura 1

Vídeo Unidad temática 4. Recuperado de Conceptos clave de apoyo a la docencia. Grado en Terapia Ocupacional 1 ( https://youtu.be/H0g5LtDXoxw)

Vídeo Unidad temática 4. Recuperado de Conceptos clave de apoyo a la docencia. Grado en Terapia Ocupacional 2 (https://youtu.be/GmFT7AINcBA)

Asignatura 2

Vídeo Unidad Temática 4 y 5: Conceptos clave de apoyo a la docencia Grado en Enfermería Metodología (https://youtu.be/q5N7aZY3tmA)

\section{ANEXO 1. Instrumentos}

Todos los instrumentos aplicados en este estudio se pueden consultar en las siguientes publicaciones:

Sáiz-Manzanares, M.C. (2018). E-project based learning en Terapia Ocupacional: una aplicación en la asignatura "Estimulación Temprana". Burgos: Servicio de Publicaciones de la Universidad de Burgos. https://www.ubu.es/catalogo-de-publicaciones/e-project-based-learning-enterapia-ocupacional-una-aplicacion-en-la-asignatura-estimulacion-temprana

Sáiz-Manzanares, M.C., Escolar-Llamazares, M.C., \& Rodríguez-Medina, J. (2019). Investigación cualitativa. Aplicación de métodos mixtos y de técnicas de minería de datos. Burgos: Servicio de Publicaciones de la Universidad de Burgos. https://www.ubu.es/catalogo-depublicaciones/investigacion-cualitativa-aplicacion-de-metodos-mixtos-y-de-tecnicas-demineria-de-datos 


\section{Apéndice 1. Algunas de las respuestas abiertas emitidas por los participantes en el estudio.}

\begin{tabular}{ll}
\hline \multicolumn{1}{c}{ Categorización } & Respuestas textuales de los estudiantes \\
\hline Mapa conceptual & "Un esquema al final del video con los \\
resumen de los & conceptos clave del tema" \\
conceptos tratados & "Incluir los concepto clave en la unidad \\
& para facilitar el aprendizaje." \\
Más ejemplos & "Más ejemplos" \\
& "Algunos ejemplos más o incluso vídeos \\
& con ejemplos de conceptos que pueden \\
& resultar difíciles de entender" \\
Más preguntas tipo & "Más preguntas tipo test" \\
test & "Más preguntas tipo test, ya que ayudan a \\
& asimilar muchos conceptos y a \\
& entenderlos"
\end{tabular}

\section{Notas biográficas}

María Consuelo Sáiz Manzanares. Licenciada en Psicología y en Ciencias de la Educación, Doctora en Psicología Educativa, Doctora en Tecnologías Industriales. Directora del GIR DATAHES y del GID B-LCS. Es Profesora Titular de Universidad en el Área de Psicología Evolutiva y de la Educación en la Universidad de Burgos. Imparte docencia en materias de evaluación e intervención que aplican métodos mixtos. Sus líneas de investigación se centran estrategias metacognitivas y aplicación de métodos mixtos y EDM que han dado lugar a numerosas publicaciones (35 artículos en revistas, 20 libros, 35 capítulos) y ponencias en congresos (700) (orcid.org/0000-0002-17362089).

María Camino Escolar Llamazares. Doctora en Psicología, Máster Universitario en Psicología General Sanitaria y Máster en Terapia de Conducta. Es Profesora Contratada Doctora del Área de Personalidad, Evaluación y Tratamiento Psicológico y Vicedecana de la Facultad de Ciencias de la Salud (Universidad de Burgos). Imparte las asignaturas "Diseño y Ejecución de la Investigación Cualitativa" y "Observación Sistemática e Investigación en Contextos Educativos". Es miembro del GIR DATAHES y del GID B-LCS (UBU). Sus investigaciones se centran en la ansiedad de evaluación, la educación para el emprendimiento y la innovación educativa y han dado lugar a numerosas publicaciones (ORCID: https://orcid.org/0000-0003-2448-0267). 